\title{
An Argus II Case Report
}

\author{
Irfan Perente, Gurkan Erdogan, Muhittin Taskapili \\ University of Health Sciences Beyoglu Eye Training and Research Hospital, Istanbul, Turkey
}

\begin{abstract}
The Argus II Retinal Prosthesis System (Second Sight Medical Products, Inc., Sylmar, CA, USA) is the first US Food and Drug Administration-approved retinal implant. It provides electrical stimulation of the retina and induces visual perception in individuals with low vision. It is used in patients with severe disease, such as retinitis pigmentosa (RP). Presently described is Argus II implantation in a patient with RP.
\end{abstract}

Keywords: Argus 2, retinal implant, retinitis pigmentosa.

\section{Introduction}

Retinal implant is recent, innovative device that offers alternative means of restoring vision in case of degenerative retinal disease. There are various implant designs that stimulate different parts of the visual pathway and the retina (I-3). The Argus II Retinal Prosthesis System (Second Sight Medical Products, Inc., Sylmar, CA, USA) was developed for incurable retinal disease with outer segment degeneration. System was approved by the US Food and Drug Administration (FDA) in 2013 for treatment in advanced stages of retinitis pigmentosa (RP). Argus II has also been implanted in patients with dry age-related macular degeneration (4).

Device is placed over the central macula and stimulates retinal ganglion cells via electrodes facing surface of the retina. It provides degree of restoration of vision by exciting secondary neurons of the retina, bypassing defunct photoreceptors. Argus II system consists of miniature video camera mounted on eyeglasses and external video processing unit that transforms visual information received by the video camera into electrical signals. Surgically implanted portion of the system comprises receiver coil that transfers digital signals to 60-electrode array implanted onto retinal surface via polymerized cable.
Presently described is case of a patient with end-stage RP who underwent Argus II implantation.

\section{Case Report}

A 43-year-old male presented at hospital with end-stage RP. The patient had late childhood-onset nyctalopia and progressive loss of peripheral vision over next few years. Finally, central vision loss occurred in both eyes. There was no systemic disease. Cataract removal had been performed on both eyes 5 years prior to presentation.

Comprehensive eye examination was performed. Visual acuity for right and left eyes was light perception with projection. Intraocular pressure was normal. Fundus examination revealed optic disc pallor, atrophic macula, and dense pattern of widespread retinal pigmentation with attenuated vessels. Optical coherence tomography revealed no vitreoretinal interface disorder or edema, but macular and foveal atrophy were present. Surgery was scheduled for the left eye. Informed consent for publication was obtained from the patient.

\section{Surgical Technique}

Implant surgery consists of 4 basic steps: preparation, extraocular placement, intraocular placement, and closure. First, 


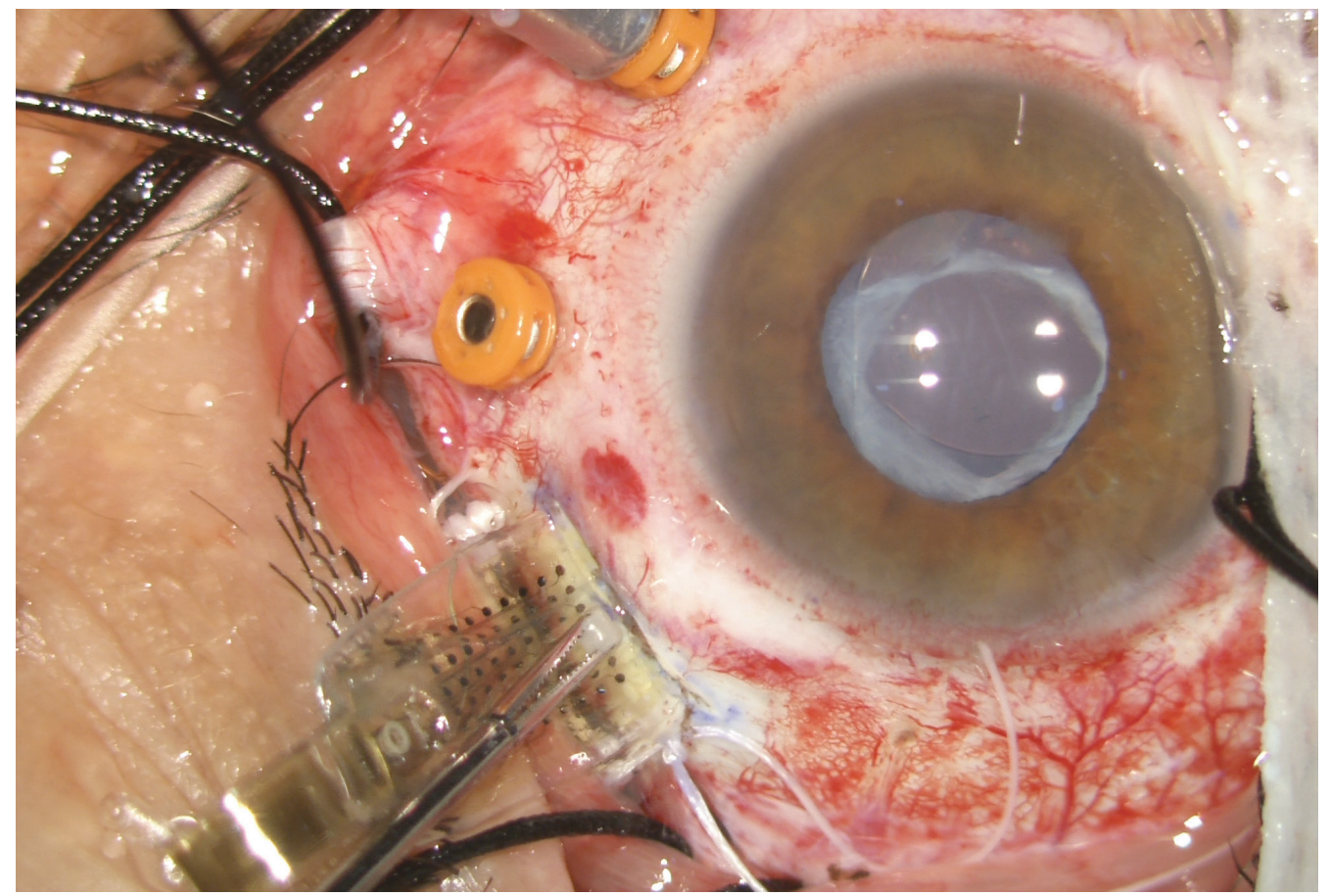

Figure I. Insertion of the electrode array through sclerotomy.

device impedances were measured. Implant was removed from tray holding scleral bands using flat forceps. Electrode array was covered with sterile silicone phaco test chamber tip to protect it from damage during surgery.

A $360^{\circ}$ peritomy, Tenon's capsule dissection, and rectus muscle isolation were performed similarly to scleral buckle procedure. External portion of prosthesis was placed on the sclera with receiving coil (antenna) under the lateral rectus muscle and metal electronics case in superotemporal quadrant. Silicone band of the device was passed under the remaining rectus muscle, secured with 5-0 nylon mattress sutures in the nasal quadrants at level of the equator. Two tips of the band were joined with silicone sleeve in superonasal quadrant. Both case and coil were fixed to the sclera by suturing anchoring tabs with $5-0$ nonabsorbable polyester suture (Dacron; Invista, Inc., Wichita, KS, USA) $6.0 \mathrm{~mm}$ posterior to the limbus in respective quadrants. This location was determined according to nomogram using axial length.

A 23-gauge complete pars plana vitrectomy was performed using triamcinolone acetonide with peripheral scleral depression. Special attention was given to complete removal of peripheral vitreous humor in superotemporal and inferonasal quadrants (quadrants for insertion of cable and tack, respectively). Distance of sclerotomy from the limbus for insertion of array was calculated using nomogram. Before sclerotomy, 30-gauge needle was inserted into the eye at that point and location of sclerotomy according to pars plana was examined with endocamera. A $5.2 \mathrm{~mm}$-wide sclerotomy was created in superotemporal quadrant at distance of 4.5 $\mathrm{mm}$ from the limbus. Electrode array and cable were inserted through sclerotomy site with 20-guage forceps (Figure I) Sclerotomy site was partially closed with 7.0 polyglactin sutures (Vicryl; Ethicon, Inc., Somerville, NJ, USA ) to limit flow. Electrode array was placed over central macula and retinal tack was loaded (Figure 2) and inserted through inferonasal sclerotomy. Electrode array was tacked to epiretinal surface while raising pressure to $60 \mathrm{mmHg}$ (Figure 3, 4). Endocamera was used to check proper placement of array over macula. Mattress sutures with 10-0 Prolene (Ethicon, Inc., Somerville, NJ, USA) were used over the cable. Closure of sclerotomies was performed with 7-0 polyglactin sutures. Tutoplast graft (Tutogen Medical, Inc., Alachula, FL, USA) was placed over the cable, suture tabs, and anterior parts of case and antenna. Closure of Tenon's capsule and conjunctiva followed.

Intraocular hemorrhage occurred postoperatively and resolved spontaneously in 3 weeks. Standard postoperative steroid and antibiotic eye drops were administered. Device was turned on and fitting was performed 3 weeks after surgery. At time of writing, the patient was 3 months post-implantation and continuing rehabilitation process.

\section{Discussion}

Argus II retinal prosthesis system was the first such device to be approved in both the US (FDA phase 4 postmarket surveillance) and Europe (phase IV, CE Marking) (5). System was evaluated in terms of clinical availability, vision resto- 


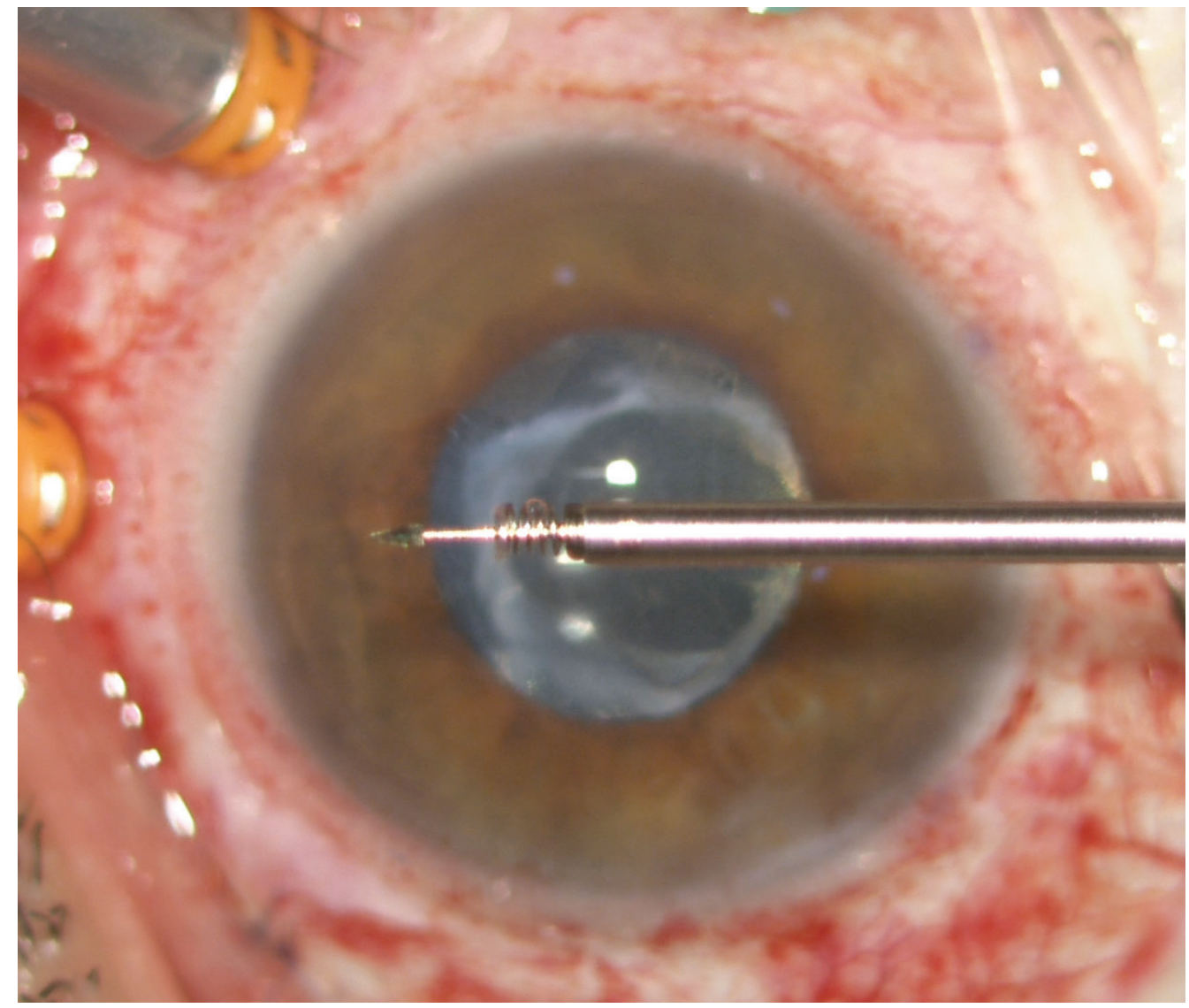

Figure 2. The loaded retinal tack.

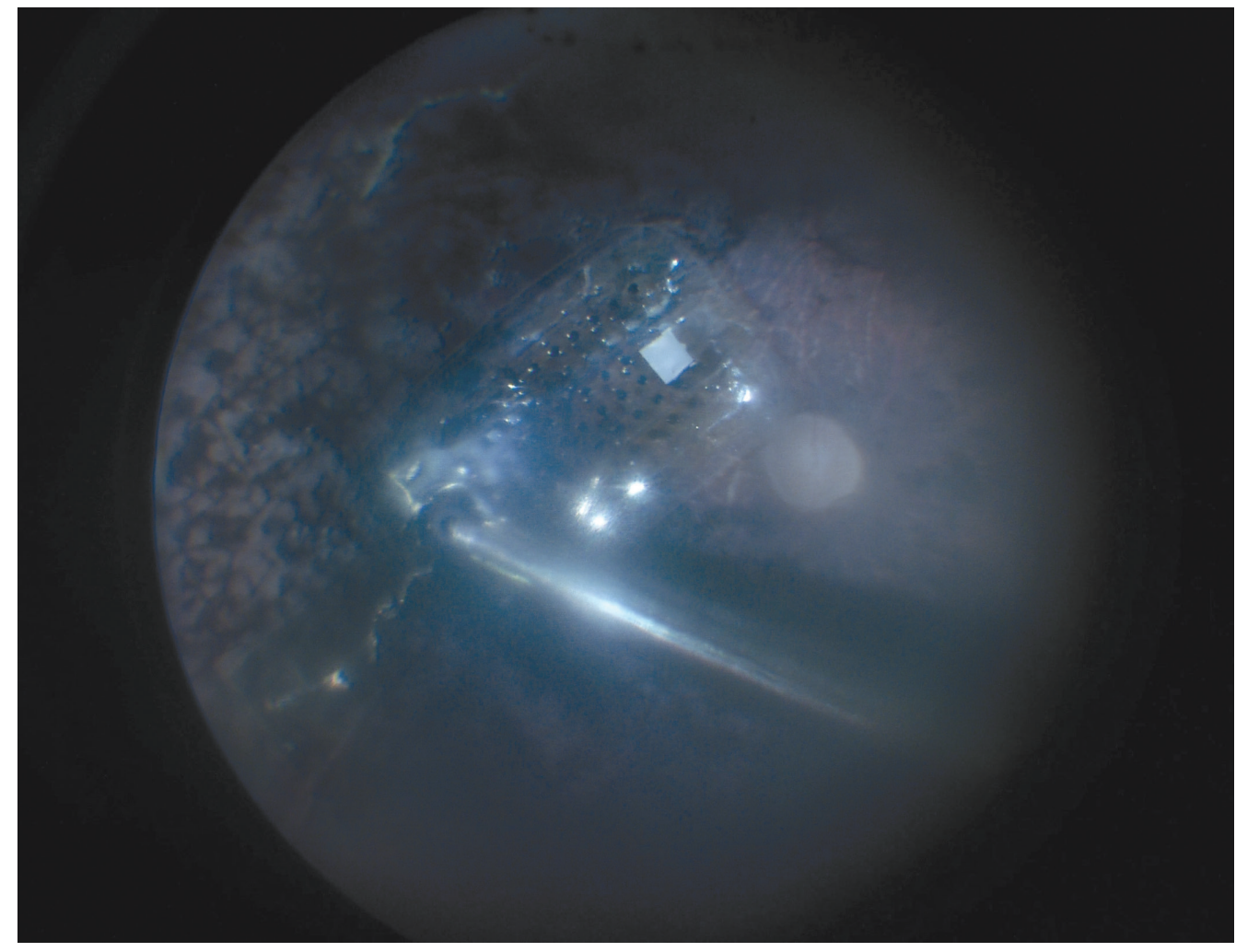

Figure 3. Tacking of the electrode array. 


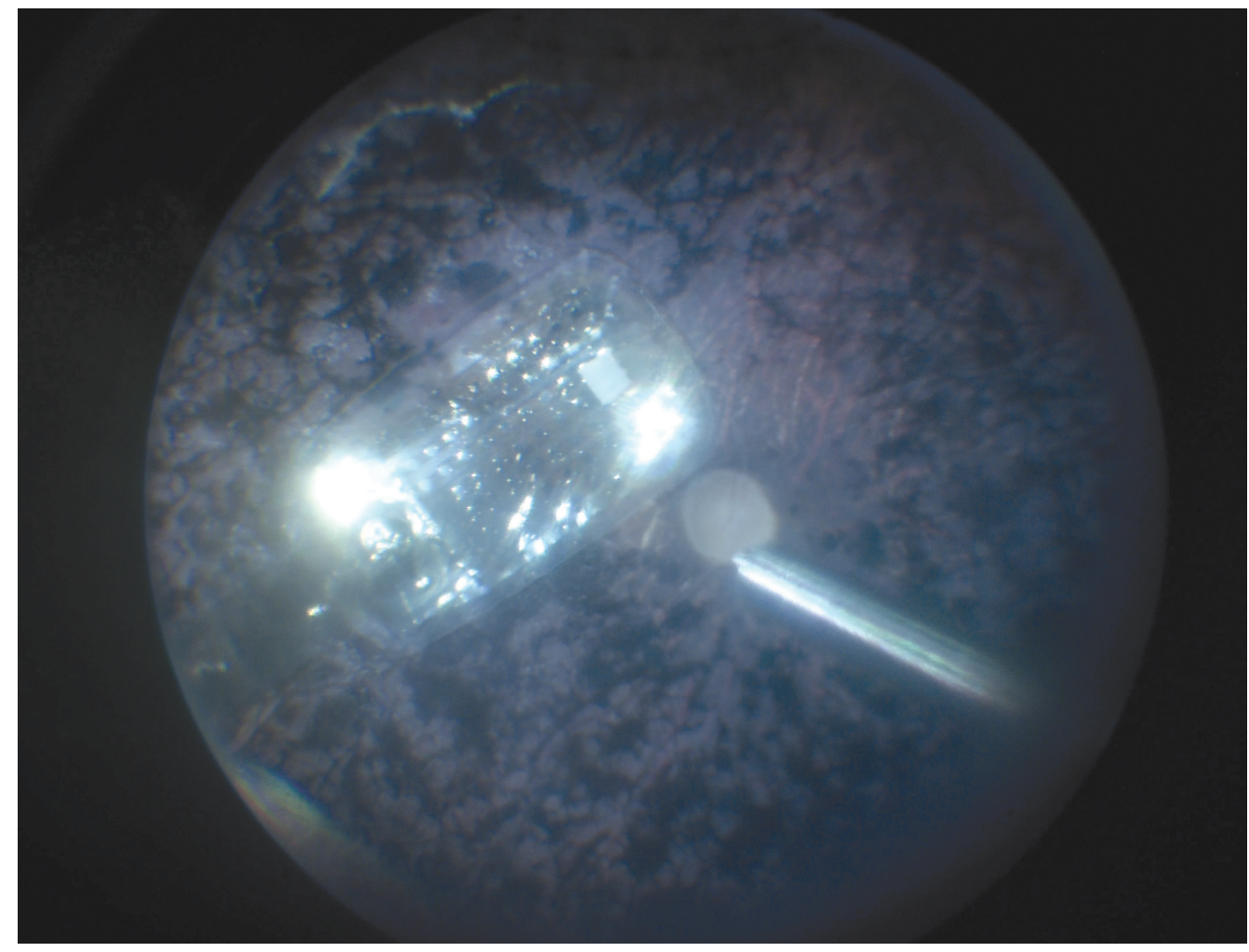

Figure 4. Positioning of the electrode array on the macula.

ration, and long-term biocompatibility, and is considered an appropriate surgical procedure for RP, a degenerative retinal disease (6). Safety and benefits of Argus II system were presented in previously published prospective, 30-patient, single-arm clinical study $(7,8)$. Argus II delivers $20^{\circ}$ visual field with external camera and 60-electrode array.

$\mathrm{RP}$ is the most common degenerative retinal disease and remains a public health problem. In advanced stages of the disease, Argus retinal implant offers promising results and seems to be effective solution. Presently described case is one of the first 10 retinal implant surgeries to be performed in Turkey.

In the present case, standard main steps of surgical procedure were followed, and additionally, the authors used endocamera during surgery. Determination of sclerotomy location for electrode array and relationship with pars plana, and position of array over the macula were examined with internal camera. It may also be used to visualize entrance of cable, the cilliary body, the pars plana, and the peripheral retina to ensure that no complications have occurred during surgery.

Vitreous hemorrhage is a potential postoperative complication of Argus II implantation (9). Mild, temporary intraocular hemorrhage occurred in our patient and resolved spontaneously in 3 weeks. We think that this bleeding was caused by large sclerotomy for electrode array and cable.
We did not observe any other postoperative complications. Montezuma et al. performed endocyclophotocoagulation of cilliary processes at the site of the intended sclerotomy superotemporally for one clock hour. They indicated that this approach could prevent cable from rubbing the cilliary body and avoid bleeding and inflammation (10).

Argus II has array of 60 electrodes, yet it does not provide very high resolution. It provides a degree of vision to those who are completely blind and improves quality of life for most patients. In the future, color vision might be enabled, resolution might be increased, and visual field might be broadened. Argus II represents a huge step forward from complete blindness and the beginning of long journey.

\section{Disclosures}

Peer-review: Externally peer-reviewed.

Conflict of Interest: None declared.

Authorship Contributions: Involved in design and conduct of the study (IP, GE, MT); preparation and review of the study (IP, GE, MT); data collection (GE).

\section{References}

I. Rizzo JF. Update on retinal prosthetic research: the Boston Retinal Implant Project. J Neuroophthalmol 201 I;31:160-8. [CrossRef]

2. Fujikado $T$, Kamei M, Sakaguchi $H$, Kanda $H$, Morimoto T, Ikuno $Y$, et al. Testing of semichronically implanted retinal prosthesis by suprachoroidal-transretinal stimulation in patients with reti- 
nitis pigmentosa. Invest Ophthalmol Vis Sci 20I I;52:4726-33.

3. Matthaei M, Zeitz O, Keserü M, Wagenfeld L, Hornig R, Post N, et al. Progress in the development of vision prostheses. Ophthalmologica 201 I;225:187-92. [CrossRef]

4. Stanga P, Jalil A, Tsamis E, Papayannis A, Dorn J, Greenberg $R$, et al. Argus II electronic epiretinal prosthesis in advanced dry AMD: Safety and feasibility study and preliminary functional results. 16th EURETINA Congress, Copenhagen; 8-II September 2016. http://www.euretina.org/copenhagen2016/downloads/EURETINACopenhagen I6_Final_Programme_Press.pdf (acces date: 8 April 2017).

5. Weiland JD, Cho AK, Humayun MS. Retinal prostheses: current clinical results and future needs. Ophthalmology 20 I I; I 18:2227-37. [CrossRef]

6. Chuang AT, Margo CE, Greenberg PB. Retinal implants: a sys- tematic review. Br J Ophthalmol 2014;98:852-6. [CrossRef]

7. Humayun MS, Dorn JD, da Cruz L, Dagnelie G, Sahel JA, Stanga PE, et al; Argus II Study Group. Interim results from the international trial of Second Sight's visual prosthesis. Ophthalmology 2012; I 19:779-88. [CrossRef]

8. Ho AC, Humayun MS, Dorn JD, da Cruz L, Dagnelie G, Handa J, et al; Argus II Study Group. Long-Term Results from an Epiretinal Prosthesis to Restore Sight to the Blind. Ophthalmology 2015; 122: 1547-54. [CrossRef]

9. Health Policy Advisory Committee on Technology. Technology Brief Update. Argus II Retinal Prosthesis System. August 2016

10. Montezuma SR, Tang PH, van Kuijk FJ, Drayna P, Koozekanani DD. Implantation of the Argus II Retinal Prosthesis in an Eye With Short Axial Length. Ophthalmic Surg Lasers Imaging Retina 20।6;47:369-7I. [CrossRef] 